\section{ESTRATEGIAS DE ADQUISICIÓN DE CONOCIMIENTO EN LOS PROCESOS DE INNOVACIÓN EMPRESARIAL}

\section{KNOWLEDGE ADQUISITION STRATEGIES IN FIRM INNOVATION PROCESSES}

\author{
Jaider Vega-Jurado, \\ Antonio Gutiérrez-Gracia \\ e Ignacio Fernández-de-Lucio \\ INGENIO-Instituto de Gestión de la Innovación \\ $y$ del Conocimiento (CSIC-UPV)
}

\begin{abstract}
The recognition of external agents as an important knowledge source to innovation processes means that the firms must face a key decision: generate in-house or acquire externally the required technological knowledge. Owing to its implications, this decision has become a very important topic between academics and practitioners of innovation, promoting the development of a body of theoretical and empirical literature about the factors and motivations which influence it. This paper provides a review of this literature via the analysis of three innovation strategies (make, buy, and cooperate) and considers the contributions coming from transaction cost theory and the firms' capabilities viewpoint.
\end{abstract}

KEY WORDS: Innovation strategies, cost transaction theory, firm capabilities.

\section{INTRODUCCIÓN}

Hasta mediados de la década de los setenta, los procesos innovadores eran explicados siguiendo básicamente un enfoque lineal que representaba las relaciones entre investigación, desarrollo, producción y marketing como unidireccionales y poco complejas. A nivel empresarial, una implicación importante de este enfoque era que circunscribía las fuentes de innovación únicamente al desarrollo de actividades de $I+D$, olvidando de esta forma el papel que desempeñan distintas modalidades de aprendizaje incremental, tales como el aprendizaje por la práctica (Arrow, 1962), el aprendizaje por el uso (Rosenberg, 1982), el aprendizaje por el error (Maidique y Zirguer, 1985) e incluso el aprendizaje a partir de los competidores (imitación).
RESUMEN: El reconocimiento de los agentes externos como una fuente importante de conocimiento para el desarrollo de los procesos de innovación ha hecho que las empresas tengan que hacer frente a una decisión importante: generar o adquirir externamente el conocimiento tecnológico que precisan. Debido a sus implicaciones, esta decisión se ha convertido en un tema de creciente interés entre los académicos y estudiosos de la innovación, propiciando el desarrollo de toda una literatura, teórica y empírica, sobre los factores y motivaciones que inciden en la misma. En este artículo se presenta una revisión de esta literatura, a partir del análisis de tres estrategias de innovación (hacer, comprar y cooperar) y considerando las aportaciones derivadas de la teoría económica de los costes de transacción y de los enfoques basados en las capacidades de la empresa.

PALABRAS CLAVE: Estrategias de innovación, teoria de los costes de transacción, capacidades de la empresa.

A partir de la década de los ochenta este tipo de enfoques fue cediendo terreno ante la emergencia de modelos interactivos que destacaban la naturaleza compleja del proceso innovador y la diversidad de las fuentes de conocimiento, tanto internas como externas, que podían ser empleadas en el mismo. De esta forma, el departamento de $I+D$, aunque importante, dejó de ser el único referente para el desarrollo de actividades innovadoras y empezó a reconocerse el valor que tenían agentes externos a la organización como fuentes de ideas innovadoras.

El pensamiento anterior se ha convertido en una de las bases para el desarrollo de la literatura reciente sobre innovación, y la contribución de las fuentes externas de conocimiento, inicialmente menospreciada, constituye hoy 
uno de los aspectos centrales a considerar en el diseño de la estrategia de innovación de las empresas. De hecho, muchas de las teorias y enfoques actuales (Teoría evolucionista, Teoría de las redes de innovación, open innovation, etc.) destacan, en mayor o menor grado, la imposibilidad de las empresas a hacer frente a los procesos de innovación por sí solas, y la consecuente necesidad de relacionarse con otros actores para llevar a feliz término su proyectos innovadores. Asimismo, la creciente externalización de las actividades de I+D y el incremento en el número de acuerdos de colaboración tecnológica entre empresas y otras instituciones, demuestran que, además de la importancia que se le ha atribuido a nivel teórico, la utilización de fuentes externas de conocimiento representa un fenómeno en crecimiento ${ }^{1}$.

No obstante, y en contraste con las dinámicas anteriores, algunos investigadores han empezado a advertir el riesgo de sobrestimar el papel de las fuentes externas de conocimiento y destacan que en muchos sectores industriales la mayor parte del esfuerzo innovador no sólo es realizado por las propias empresas sino que además se desarrolla en el interior de las mismas (Nelson, 2000). Los estudios realizados por Oerlemans et al. (1998) en Holanda y por Freel (2003) en el Reino Unido, muestran que los recursos internos de la empresa constituyen el principal determinante de su desempeño innovador, y que, por ejemplo, el establecimiento de redes con agentes externos ejerce un efecto limitado. Adicionalmente, algunos autores han llegado incluso a sugerir que en su deseo por externalizar las actividades de I+D las empresas pueden estar debilitando sus competencias nucleares (Coombs, 1996).

Como corolario a los planteamientos anteriores, la búsqueda y adquisición de conocimiento tecnológico ha emergido como un aspecto clave y complejo dentro de la estrategia de innovación empresarial ${ }^{2}$. Las empresas no sólo tienen que decidir entre generar internamente el conocimiento que requieren o adquirirlo de fuentes externas, sino además seleccionar el mecanismo más adecuado que le permita acceder a dicho conocimiento. Estos mecanismos han sido referidos en la literatura como estrategias de innovación o estrategias tecnológicas ${ }^{3}$, y su análisis se ha convertido en un tema de gran interés entre los académicos de la innovación. ¿Cómo las empresas pueden adquirir el conocimiento tecnológico que precisan? y ¿qué factores influyen en la decisión empresarial sobre que estrategia de innovación emplear? son algunas de las preguntas que surgen como elementos relevantes de estudio.

Este artículo analiza precisamente cómo estas cuestiones han sido abordadas en la literatura, destacando tanto los enfoques conceptuales empleados para el estudio de las mismas, como los principales resultados empíricos obtenidos. El objetivo es presentar una revisión crítica de los enfoques utilizados, señalando sus diferencias, fortalezas y debilidades, así como las principales líneas de investigación que permanecen aún abiertas. Para ello el artículo se estructura de la siguiente forma: en la sección 2 se presenta una descripción general de las perspectivas teóricas a partir de las cuales se ha abordado el análisis de las estrategias de innovación; en la sección 3 se describen algunos de los estudios empíricos realizados hasta la fecha y en la sección 4 las principales conclusiones.

\section{Estrategias dE INNOVACIÓN}

Las empresas tienen distintas opciones para adquirir el conocimiento tecnológico que necesitan para llevar a cabo sus procesos innovadores. Por ejemplo, una primera estrategia es optar por la generación de conocimiento, a través del desarrollo interno de actividades de I+D, confiando de esta forma en las capacidades propias de la organización. Esta estrategia ha sido referida generalmente en la literatura como la decisión de Hacer. Una segunda alternativa es adquirir la tecnología externamente, a través de transacciones de mercado. En este caso las empresas tienen dos opciones principales: adquirir conocimiento tecnológico incorporado en bienes o activos (bienes de capital, herramientas o inputs del proceso productivo como materiales y componentes) o adquirir conocimiento tecnológico no incorporado en ningún elemento material tangible, bien sea, subcontratando I+D o a través de licencias de patentes. Todas estas alternativas han sido asociadas con la decisión de Comprar. Por último, una tercera forma de obtener y desarrollar tecnología es a través del establecimiento de acuerdos de colaboración con otras empresas o instituciones, es decir emplear la estrategia de Cooperar.

Si bien es cierto que bajo las denominaciones genéricas de Hacer, comprar y cooperar se pueden encontrar diferentes mecanismos de adquisición de conocimiento tecnológico, 
esta tipología ha sido la base para el análisis de las estrategias de innovación en la teoría económica de la empresa. Mientras que la primera estrategia se fundamenta en el uso de las fuentes internas de conocimiento, las otras dos están directamente relacionadas con la utilización de fuentes externas. En este sentido, la diferencia fundamental entre comprar y cooperar, es que mientras que en la compra se desarrolla una relación unilateral (dinero es intercambiado por resultados de I+D), en la cooperación cada parte contribuye a la relación proporcionando conocimiento de valor (Croisier, 1998).

El análisis de estas estrategias no sólo permite conocer mejor los procesos de generación y difusión de conocimientos asociados a las actividades innovadoras, sino que además facilita la identificación de los mecanismos de vinculación que deben ser fomentados a través de las políticas de innovación. Preguntas como: ¿la externalización de las actividades de I+D constituye una estrategia eficaz para el desempeño innovador de la empresa?, ¿deberían las políticas de innovación basarse en la promoción de la cooperación entre empresas y otras instituciones? y, en caso afirmativo, ¿cuáles son los socios más importantes?, son sólo algunas de las cuestiones que emergen del estudio de este tema y cuyas respuestas revisten especial importancia tanto en el ámbito de la gestión empresarial como en al ámbito de las políticas públicas.

Teniendo en cuenta lo anterior, no es de extrañar que una de las primeras aproximaciones al estudio de estas estrategias se derive precisamente de la teoría económica, en concreto de la teoría de los costes de transacción (TCT). Esta teoría, cercana a la corriente económica neoclásica, se ha consolidado como uno de los enfoques más importantes para analizar lo que se ha denominado las "fronteras de la empresa", es decir, la decisión empresarial entre el desarrollo interno o externo de una actividad.

El análisis de las estrategias de innovación se ha enriquecido también gracias a las aportaciones derivadas de la literatura relacionada con las capacidades de la empresa. Estas aproximaciones son más recientes que la TCT y sus fundamentos se encuentran más en línea con los de la teoría evolucionista.

A continuación se explican con mayor detalle los fundamentos de cada uno de estos enfoques.

\subsection{El análisis de las estrategias de innovación desde el prisma de la teoría de los costes de transacción (TCT)}

La TCT, ejemplificada principalmente por las contribuciones de Williamson (1981, 1985), sugiere que existen dos mecanismos o estructuras de gobierno alternativas para llevar a cabo una transacción: a través del mercado o internamente en la empresa. Mientras que en el mercado, la transacción es regulada por el sistema de precio, en la empresa (ó jerarquías en términos de Williamson) la autoridad se convierte en el factor más importante. Según la TCT la elección entre una u otra forma de gobierno es una decisión que se basa en la consideración de los costes (ex ante y ex post) asociados a cada una de las alternativas, por lo que su objetivo se centra en identificar las fuentes de dichos costes.

Aunque Williamson no estudió directamente las estructuras de transacción relacionadas con la adquisición de conocimiento tecnológico, la dicotomía entre mercadojerarquía ha sido utilizada por otros investigadores como base para el análisis de las estrategias de innovación. De esta forma, los principios que emplea la TCT para predecir cuál es la estructura de gobierno más eficiente (menor coste) para llevar a cabo una transacción, han sido utilizados para explicar los factores que determinan la decisión entre externalizar o generar internamente el conocimiento tecnológico. Estos principios se derivan directamente de los atributos o características de la transacción, entre los cuales se destacan el grado de incertidumbre y la especificidad de los activos ${ }^{4}$.

La incertidumbre hace referencia tanto a los cambios potenciales en el entorno, como a la imprevisibilidad del comportamiento del socio. Cuanto mayor es la incertidumbre más difícil es realizar una descripción completa de la transacción a través de un contrato y por lo tanto se requerirán negociaciones posteriores para adaptarse a las condiciones no previstas. De forma similar, cuanto mayor es la especificidad de los activos objeto de la transacción, más necesidad habrá de establecer cláusulas de salvaguardias que permitan disminuir el riesgo de abandono por la otra parte ${ }^{5}$. Por lo anterior y debido al oportunismo y a la racionalidad limitada de los agentes, la TCT sugiere que cuando las transacciones tienen un alto grado de incertidumbre o involucran activos específicos, los costes de 
intercambio en los mercados se incrementan, motivando a la empresa a producir internamente. Adicionalmente, en estas condiciones la empresa, como estructura de gobierno, tiene una mayor capacidad de coordinación, debido a que las disputas son resueltas a través de la autoridad en lugar de la negociación.

Siguiendo este marco conceptual, la estrategia de hacer resultaría más eficiente en aquellos casos en los cuales la empresa requiere un conocimiento tecnológico muy específico, derivado, por ejemplo, de actividades complejas de I+D. Actividades de este tipo están expuestas a una alta incertidumbre, no sólo tecnológica (¿qué resultados se alcanzarán?), sino también temporal (¿cuándo se obtendrán?) y comercial (¿qué retornos económicos se obtendrán?), la cual supone una gran dificultad para describir adecuadamente el objeto de la transacción en un contrato y transferir de una forma eficiente el conocimiento entre un proveedor y un receptor (Croisier, 1998). Ante esta dificultad se requerirán continuas negociaciones para adaptar el comportamiento de las partes durante las diferentes etapas del proceso, por lo que los costes de negociación superarán ampliamente los costes de coordinación y desarrollo interno.

Pero, el uso de la TCT no se limita al análisis de las estrategias anteriormente mencionadas. Varios investigadores (Tyler y Steensma, 1995; Veugelers, 1998; Oerlemans y Meeus 2001) han señalado que este enfoque constituye también una herramienta útil para analizar la estrategia de cooperación, contemplándola como una forma de gobierno intermedia entre las estructuras alternativas de hacer (jerarquia) y comprar (mercado) 6 . En este sentido, se ha sugerido que la cooperación es una forma hibrida de organización, que permite el acceso al conocimiento especializado que poseen otras instituciones -aspecto claramente restringido en las transacciones jerárquicas- pero a un coste menor comparado con las transacciones del mercado (Pisano, 1989, 1990).
Una representación de las estrategias tecnológicas bajo el prisma de la TCT puede ser la esquematizada en la Figura 1. La línea horizontal representa el "continuo" formado por el mercado y la jerarquía. En los extremos de dicha línea, y por lo tanto más asociadas con estas formas puras de gobierno, se encuentran las estrategias de comprar y hacer, mientras que la cooperación se ubica en el medio de las mismas. Adicionalmente, también pueden distinguirse diferentes mecanismos asociados a cada estrategia, cuya ubicación con respecto a la línea horizontal indica cuanto se acercan o se alejan de una forma de gobierno específica. La realización de actividades internas de $I+D$, por ejemplo, puede llevarse a cabo de forma centralizada o descentralizada (a través de los diferentes departamentos o unidades de negocio de la empresa), siendo la primera opción la estructura de gobierno jerárquica por excelencia. Asimismo, la cooperación puede establecerse a través de mecanismos basados en la participación patrimonial (joint ventures) o a través de acuerdos contractuales; los primeros con rasgos más cercanos a las jerarquías y los segundos con características más cercanas al mercado. Por último, dentro de la estrategia de comprar pueden distinguirse tres mecanismos: la subcontratación de actividades de I+D, el licenciamiento de tecnología y la adquisición de conocimiento incorporado en maquinaria y equipos. Aunque estos tres últimos mecanismos representan estructuras de gobierno basadas en el mercado, la subcontratación de I+D está un poco más cercana al polo de la jerarquía debido a que encierra una mayor posibilidad de control por parte del contratista.

La clasificación anterior, si bien no es exhaustiva, incluye algunas de las estrategias de innovación más importantes. En la misma se contemplan no sólo las estrategias asociadas a la I+D o a la adquisición de tecnología a través de las licencias de patentes, sino que además se incluye la compra de maquinaria y equipo, cuyo valor como input en los procesos de innovación no puede ser desatendido. 


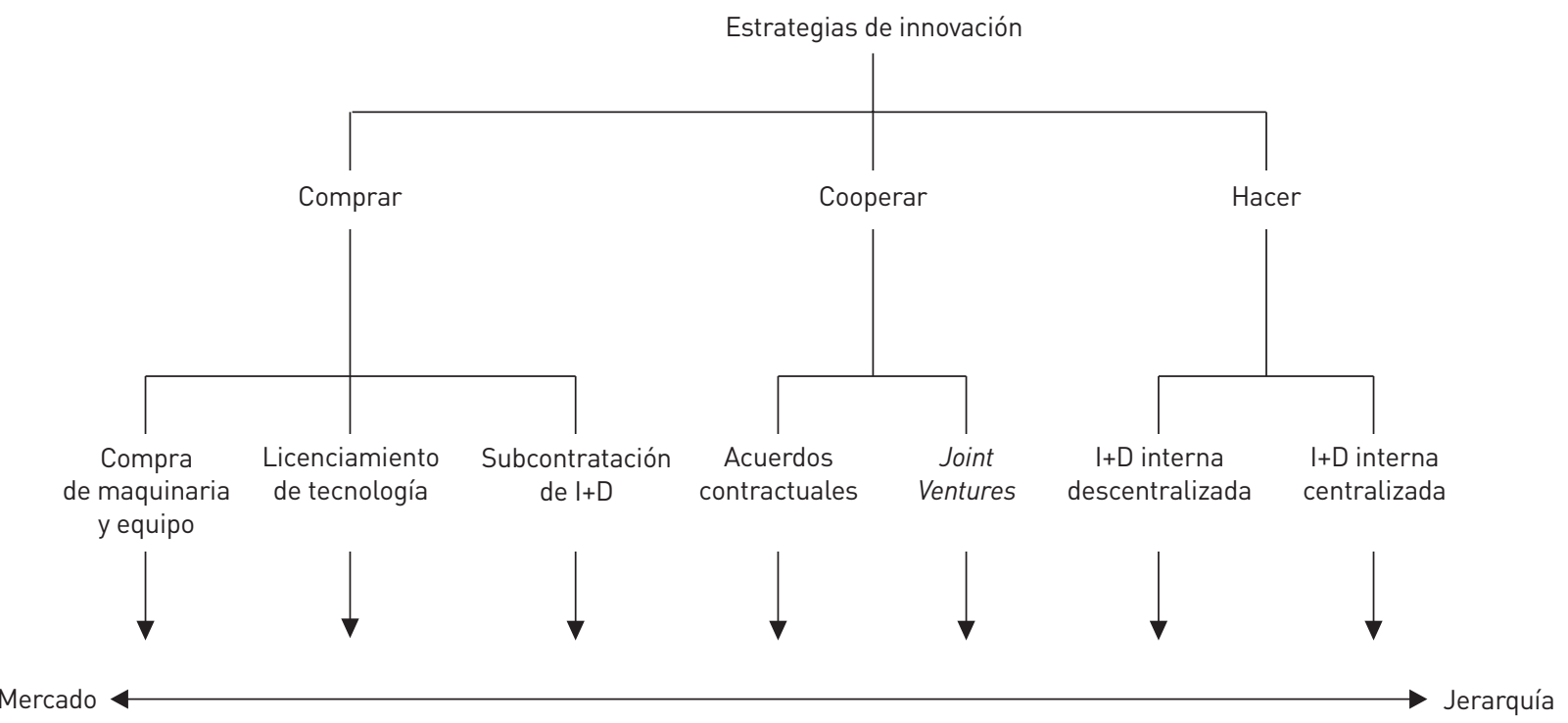

Fuente: Adaptado de Croisier (1998, p. 292).

\subsection{El análisis de las estrategias de innovación desde la perspectiva de las capacidades de la empresa}

A pesar de que la TCT ofrece argumentos importantes con relación a los atributos de la transacción que influyen en la elección de la estrategia de innovación, su lógica, basada en la minimización de costes, no captura muchas de las ventajas estratégicas asociadas al uso de las fuentes externas de conocimiento y por ende a la adopción de estrategias como comprar y cooperar. De hecho, varios autores han tildado esta aproximación de superficial. Chesnais (1996), por ejemplo, realiza una crítica tanto al enfoque de los costes de transacción en general, como a la proposición particular de que las alianzas representan un estado "intermedio" entre el continuo conformado por el mercado y la jerarquía. Según su parecer, los acuerdos colaborativos deberían ser analizados como un fenómeno en sí mismo, y las redes tratadas como una forma distinta de organización económica usada para intercambiar recursos y crear activos de valor.

Oerlemans y Meeus (2001) señalan igualmente que la TCT ofrece una explicación parcial al fenómeno de la cooperación, debido a que se centra fundamentalmente en los rasgos de la actividad organizacional (por ejemplo, la or- ganización de las transacciones) y presta poca atención a los recursos implicados durante el proceso. En este sentido Tyler y Steensma (1995) destacan que la cooperación tecnológica no es sólo un modo de coordinación de las transacciones, sino que además, es una actividad donde los recursos y el know-how de la empresa son factores que se necesitan y que al mismo tiempo se desarrollan.

La forma con la que se trata las estrategias de innovación en el marco de la TCT y la parcialidad de sus supuestos no es, sin embargo, un hecho que deba sorprender teniendo en cuenta que sus principios son coincidentes con los de la teoría económica neoclásica. El énfasis de esta teoría recae sobre el agente individual y la predicción de la competencia perfecta como el estado más eficiente de la economía, un marco en el cual las relaciones entre los agentes no se presentan como un elemento importante. Es en este punto donde la visión de las capacidades de la empresa aparece como un enfoque de análisis complementario. Dentro de este marco se destacan la teoría basada en los recursos (TBR) (Wernerfelt, 1984) y el enfoque de las capacidades dinámicas (Teece et al., 1997), las cuales, aunque pertenecen al campo de la gestión empresarial, se han desarrollado gracias a las contribuciones de la teoría económica evolucionista. 
La TBR tiene como supuesto fundamental el carácter heterogéneo de las empresas, derivado de la posesión de un conjunto único de recursos (tangibles e intangibles) que han sido desarrollados a lo largo de su historia. Estas diferencias de recursos se mantienen en el tiempo y la explotación de los mismos es la que le otorga a la empresa una ventaja competitiva sostenible (Wernerfelt, 1984) Por su parte, el enfoque de las capacidades dinámicas, aunque reconoce la importancia de los recursos internos de la empresa, subraya que la verdadera ventaja competitiva se deriva de la capacidad que tenga la organización para generar nuevos recursos en función de las demandas del entorno. Para ello es imprescindible que la dirección de la empresa tenga la habilidad para coordinar y disponer tanto de capacidades internas como externas (Teece et al., 1997).

Siguiendo estas perspectivas, la lógica detrás de la elección de una estrategia de innovación no está basada en la disminución de costes, sino en la posibilidad de acceder o generar recursos que permitan desarrollar una ventaja competitiva sostenible. Por ello, en lugar de considerar los atributos de la transacción (conocimiento tecnológico), estas perspectivas centran su atención en el análisis de las características tanto de la empresa como del proveedor como factores determinantes de la estrategia de innovación. En esta línea el argumento general es que las empresas tienden a producir bienes que están más cercanos a su área de negocio o que están relacionados con bienes que ya producen. De esta forma, cuanto mayor es la capacidad que tiene una empresa con relación a la producción de un bien, mayor será la internalización del mismo y, contrariamente, cuanto mayor es la capacidad del proveedor, más probable es que dicho bien sea adquirido externamente. En el caso en el que la empresa y el proveedor posean una capacidad relativamente grande para la producción de dicho bien, la empresa puede estar motivada tanto a aprovechar su propio conocimiento como a aprender de la experiencia del proveedor.

Lo anterior, por ejemplo, supone que una empresa escogerá la estrategia de hacer si tiene fuertes capacidades en el desarrollo de actividades de I+D y además el conocimiento que requiere está fuertemente relacionado con el núcleo de su negocio. Por el contrario, optará por la decisión de comprar o cooperar si el conocimiento que requiere está débilmente relacionado con sus capacidades tecnológicas y/o el proveedor pueda ofrecerle recursos complementarios que faciliten el desarrollo de nuevas capacidades.

El concepto de recursos complementarios ocupa un lugar central en estas perspectivas, hasta el punto que se reconoce como motivación principal para el uso de fuentes externas de conocimiento la búsqueda de complementariedades tecnológicas. Siguiendo esta línea, se argumenta generalmente que dada la naturaleza compleja de muchas de las tecnologías y productos actuales, es muy difícil para una empresa desarrollar y mantener por si misma todas las capacidades necesarias para hacer frente a las actividades innovadoras. En este sentido, las empresas recurren a las fuentes externas de conocimiento principalmente como una estrategia para apalancar sus competencias internas y aprovechar las economías de alcance (sinergias).

Este último planteamiento constituye una diferencia importante con relación a la TCT. Al asumir la complementariedad tecnológica como el motivo principal para el uso de fuentes externas de conocimiento, el proceso adquiere igual o más importancia que el resultado obtenido. En otras palabras, el valor de estrategias como comprar o cooperar radica no sólo en el resultado final, sino en el aprendizaje generado a través de la misma.

Como puede observarse, los enfoques que se basan en una visión estratégica de la empresa contemplan la utilización de fuentes externas de conocimiento más como una oportunidad para aprender que como una alternativa para la disminución de costes. Esta distinción es relevante debido a que intrinsicamente destaca la posibilidad de desarrollar nuevas competencias organizacionales a partir de los procesos de aprendizaje derivados de la interacción con agentes externos.

Sin embargo, a pesar de las diferencias existentes entre ellas, la teoría de los costes de transacción y las perspectivas de la gestión estratégica de la empresa, representan, más que aproximaciones contrarias, enfoques complementarios para el análisis de un mismo fenómeno. De hecho, tal como se ha puesto de manifiesto en numerosos estudios empíricos, las motivaciones tácticas y estratégicas pueden llegar a entrecruzarse en la estructura de las decisiones empresariales, aunque estas últimas tiendan a ser, por lo general, más relevantes (Sakakibara, 1997; Mariti and Smiley, 1983; Veugelers, 1998; Hagedoorn, 2002). 


\section{Estudios EMPíricos SOBRE LAS ESTRATEgIAS DE INNOVACIÓN}

Existe un número considerable de estudios empíricos que abordan el análisis de las diferentes estrategias de innovación aquí mencionadas, aunque en muchos casos no sean denominadas como tal. Una parte considerable de estos estudios se ha focalizado en la identificación de los factores que influyen en la adopción de cada estrategia y, más recientemente, en el análisis de las relaciones de sustitución o complementariedad existentes entre las mismas.

\subsection{Análisis de los factores asociadas al uso de cada estrategia}

Uno de los temas que más interés ha despertado tanto en la literatura económica como en la literatura de la gestión empresarial ha sido el análisis de los factores que influyen en la elección entre las diferentes estrategias de innovación. Las primeras investigaciones sobre este aspecto se centraron casi de forma exclusiva en el análisis de los determinantes de las actividades internas de I+D, es decir, en el análisis de la estrategia de hacer. La investigación empírica desarrollada en esta línea tuvo como punto de partida la verificación de las hipótesis clásicas schumpeterinas que relacionaban un mayor tamaño de la empresa y una estructura del mercado monopólica con un mayor esfuerzo en $\mathrm{I}^{+} \mathrm{D}^{7}$. Posteriormente, con el reconocimiento del valor de las fuentes externas de conocimiento y el crecimiento tanto de la colaboración tecnológica como de la externalizacion de la I+D, los investigadores empezaron a explorar los determinantes de otras posibles estrategias de innovación empresarial. Mowery (1983) y Pisano $(1989,1990)$ fueron algunos de los primeros autores en analizar el diseño de la estrategia de innovación como un problema de elección entre alternativas distintas y en identificar los factores que influyen en dicha elección. Estos autores siguieron básicamente el enfoque de la TCT, por lo que centraron su análisis en los atributos del conocimiento tecnológico, particularmente en los atributos de las actividades de I+D. Mowery por ejemplo, identificó la especificad e interdependencia de la I+D como factores clave que influyen en su externalización. En concreto, este autor señaló que cuando la $I+D$ es de naturaleza genérica (bajo nivel de especificidad) o está focalizada en aspectos aislados o fácilmente separables de las otras funciones organizacionales (p.e. producción, marketing, etc.) la com- pra es mucho más probable como estrategia de innovación que el desarrollo interno. Pisano, por su parte, señaló que ante la posibilidad de que la transacción relacionada con la adquisición de conocimiento tecnológico requiriese negociaciones más intensas, la empresa encontraría más atractivo el desarrollo interno de la I+D en lugar de su externalizacion.

Una aplicación más rigurosa de la TCT al análisis de las estrategias de innovación es presentada por Croisier (1998). Este autor analiza cómo la incertidumbre, especificad y frecuencia de los proyectos de I+D inciden sobre la elección de su estructura de gobierno. Sus resultados concuerdan con los principios generales de la TCT, indicando que cuanto mayor es la incertidumbre, especificad y frecuencia del proyecto de $I+D$, más cercano estará de realizarse a través de mecanismos cercanos a la jerarquía (hacer).

Por otra parte, existe también una corriente de estudios que, siguiendo las perspectivas de las capacidades de la empresa, centran su atención en el análisis de los atributos de la empresa como determinantes de la estrategia de innovación. En este grupo se destacan trabajos como los de Lowe y Taylor (1998), Miotti y Sachwald (2003) y Belderbos et al. (2004), los cuales sugieren que la adquisición externa de conocimiento, ya sea a través de la compra o de la cooperación, es una estrategia que depende de la existencia de capacidades complementarias en el interior de la empresa. Estas capacidades incluyen no sólo las tecnológicas, sino también las capacidades organizacionales no relacionadas con $l a \mathrm{I}+\mathrm{D}$, tales como el marketing, la manufactura, el poder de mercado e incluso la imagen.

A pesar de que la TCT y las perspectivas de las capacidades de la empresa inciden en aspectos diferentes, se ha demostrado que su integración puede ofrecer un marco analítico más robusto. Oerlemans y Meeus (2001), por ejemplo, conjugan en su investigación principios propios de la TCT (frecuencia, especificidad e incertidumbre) y de la teoría basada en recursos para analizar los determinantes de la cooperación en I+D. Sus resultados empíricos demuestran que cuando se incluyen indicadores derivados de ambas perspectivas, los modelos analíticos incrementan su poder explicativo. Un estudio similar, desarrollado en el contexto español, es presentado por Beneito (2003), quien emplea conjuntamente indicadores relacionados con los atributos 
de las actividades de I+D y con las capacidades de la empresa para analizar la elección entre las estrategias hacer y comprar.

\subsection{Análisis de las relaciones entre las estrategias de innovación}

Además del análisis de los factores que influyen en la adopción de las estrategias de innovación, otro tema que ha sido abordado en la literatura es el concerniente a las relaciones existentes entre las mismas. Hay que señalar que los primeros estudios realizados sobre este tema seguían la tradición derivada de la TCT, la cual sugería una completa sustitución entre las diferentes estrategias de innovación, por lo que por motivos de costes y riesgos la empresa debía elegir entre adoptar una u otra. De esta forma, se consideraba que lo que afrontaba la empresa era básicamente un problema de selección entre alternativas sustitutivas, es decir, decidir entre hacer, comprar o cooperar como mecanismo para adquirir tecnología. Dentro de este marco conceptual, no se contemplaba la existencia de una relación entre las estrategias de innovación, más allá de su perfecta sustitución, y los análisis se centraban en determinar las condiciones bajo las cuales una estrategia era preferible sobre otra.

Mowery (1983), fue quizás el primer autor en sugerir la existencia de posibles relaciones entre las estrategias $h a-$ cery comprar. En su estudio sobre los factores que influyen en la externalizacion de las actividades de $I+D$, este autor no sólo señaló elementos consistentes con el enfoque de la TCT, sino que además indicó que cuanto mayor es la capacidad tecnológica de una empresa más probable es que ésta emplee la adquisición externa de tecnología como estrategia de innovación. Esta última argumentación va más allá de los fundamentos de la TCT e incluso la contradice en algunos aspectos. Si se tiene en cuenta que el desarrollo de actividades internas de I+D es una de las fuentes para el desarrollo de las competencias tecnológicas de la empresa, lo anterior supondría que las estrategias de hacery comprar, más que opciones alternativas, pueden llegar a ser elementos complementarios en la estrategia de innovación empresarial. Esta relación está más cercana a los enfoques basados en las capacidades de la empresa, lo cuales desatacan la complementariedad tecnológica como la motivación principal detrás de la adquisición externa de conocimiento.
El trabajo de Mowery abrió las puertas para que se estudiara no sólo los beneficios de cada estrategia particular, sino las potencialidades asociadas a su combinación dentro de la estrategia de innovación global de la empresa. Esta corriente tuvo un fuerte impulso gracias a la publicación del trabajo seminal de Cohen y Levinthal (1990) sobre la capacidad de absorción. Estos autores señalaron que las actividades internas de I+D cumplen un doble papel. Por una parte, constituyen una fuente de conocimiento importante para la generación de ideas innovadoras y, por otra, incrementan la capacidad de la empresa para identificar, asimilar y explotar el conocimiento disponible fuera de sus fronteras, es decir, incrementan su capacidad de absorción. Partiendo de este concepto, se han desarrollado diversos estudios empiricos que analizan las relaciones existentes entre el know-how interno y externo o, en términos estratégicos, las relaciones entre las decisiones de hacer y comprar. Arora y Gambardella $(1990,1994)$, por ejemplo, encontraron que las grandes empresas con una mayor base de conocimiento son más activas en la búsqueda y adquisición de tecnología externa, mientras que Veugerlers (1997) encontró evidencia de una relación en sentido inverso en la medida en que la adquisición externa de conocimiento estimulaba, bajo ciertas condiciones, el desarrollo de actividades internas de $\mathrm{I}+\mathrm{D}$.

En términos generales, existe evidencia empírica consistente a favor del papel que ejerce el conocimiento interno en la identificación y adquisición del conocimiento externo e, inversamente, del estímulo que otorga la adquisición externa de tecnología al desarrollo de actividades internas de I+D. Estos hallazgos corroboran la posibilidad que tiene la empresa para adoptar conjuntamente diversos mecanismos orientados a la obtención de tecnología, en otras palabras, que las estrategias hacer, comprar y cooperar coexisten en la estrategia global empresarial.

Los resultados anteriores han llevado a la emergencia de una nueva línea de investigación centrada en el análisis de las posibles complementariedades entre las diferentes estrategias de innovación. Dicha complementariedad, implica mucho más que la adopción conjunta de diferentes estrategias; supone la existencia de un efecto sinérgico entre ellas, en la medida en que la adopción de una incrementa el retorno marginal de la otra (Milgrom y Roberts, 1990), por ejemplo, si el desarrollo de actividades internas de I+D (hacer) incrementa la efectividad del 
conocimiento adquirido externamente para el desarrollo de innovaciones.

La investigación sobre este último tema es aún incipiente y se enfrenta a dificultades metodológicas considerables (Athey and Stern, 1998). Adicionalmente, los pocos estudios realizados hasta el momento han llegado a resultados contradictorios. Cassiman y Veugelers (2006), por ejemplo, encontraron que las actividades internas de I+D y la adquisición externa de conocimiento tenían efectos complementarios sobre el desempeño innovador de la empresa, mientras que Laursen y Salter (2006) encontraron efectos de sustitución entre la intensidad en I+D y el uso de fuentes externas de conocimiento.

\section{Conclusiones}

A pesar de las notables diferencias existentes entre la teoría de los costes de transacción y los enfoques basados en las capacidades de la empresa, se ha demostrado que, más que sustitutas, pueden llegar a ser aproximaciones complementarias para el análisis de las estrategias de innovación empresarial. La integración de estos enfoques en un marco de análisis común, ofrece a los investigadores la posibilidad de considerar un mayor número de factores como posibles determinantes de la estrategia de innovación empresarial y alcanzar de esta forma un mayor poder explicativo. Es más, la lógica de la TCT basada en el análisis de los atributos del conocimiento tecnológico y la de los enfoques basados en las capacidades de la empresa parecen reforzarse mutuamente, cubriendo los vacíos existentes en cada una de ellas.

Los trabajos empíricos desarrollados en los últimos años han permitido mejorar nuestro entendimiento sobre los factores y motivaciones que influyen en la elección de las diferentes estrategias de innovación y han demostrado que las empresas usualmente combinan el desarrollo interno con la adquisición externa de conocimiento tecnológico. En este sentido, la preocupación fundamental de los investigadores parece orientarse ahora hacía el análisis de las complementariedades existentes entre las estrategias hacer, comprar y cooperar, en cuanto a su efecto sobre el desempeño innovador de la empresa. Avanzar en esta línea reviste gran interés, no sólo desde el punto de vista teórico, sino también a nivel práctico, dada sus implicaciones en el ámbito de la gestión empresarial y en el ámbito de las políticas públicas.

\section{NOTAS}

Recibido: 15 de abril de 2008

Aceptado: 30 de mayo de 2008
1 En la mayor parte de los paises de la OECD, los gastos empresariales en I+D externa han mostrado un incremento considerable, incluso superior al exhibido por los gastos totales en I+D o en innovación. En paises como el Reino Unido o Alemania ha sido tal este crecimiento que el peso relativo de la I+D externa en la estructura del gasto total en $I+D$, ha llegado casi a duplicarse en un período de 10 años (Howells, 1999; Bönte, 2003). Asimismo, Hagedoorn (2002) presenta un interesante trabajo donde analiza la evolución de los acuerdos formales de I+D durante los últimos 40 años utilizando como fuente de información la base de datos MERIT-CATI. Dentro de los resultados de este trabajo se destaca el importante crecimiento en el número de dichos acuerdos, los cuales pasaron de ser casi 10 por año en la década de los sesenta a ser más de 600 en el año de 1995.

2 El término de conocimiento tecnológico es usado en este artículo en un sentido amplio e incluye el conocimiento científico básico, el conocimiento aplicado, el desarrollo experimental, y no sólo el conocimiento basado en la I+D.

3 En la literatura económica se tiende a emplear indistintamente estos términos, aunque en la literatura de la gestión empresarial algunos autores 
(Martínez, 1989 y Benavides, 1998) han señalado los riesgos que dicha confusión puede suponer para el diseño de estrategias organizacionales. Este artículo sigue la tradición de la literatura económica $y_{1}$ a menos que se especifique lo contrario, estrategias de innovación y estrategias tecnológicas harán referencia a los mecanismos que emplea la empresa para generar y/o adquirir el conocimiento necesario para llevar a cabo proyectos innovadores.

4 Si bien la incertidumbre y la especificidad de los activos no son las únicas características a tener en cuenta, Williamson las reconoce como las más importantes al momento de analizar las condiciones que llevan a la sustitución de los mercados como estructura de gobierno de la transacción.

5 Un activo específico es aquel que pierde mucho de su valor si es empleado en una actividad diferente a aquella para la cual fue diseñado, o si es empleado por un usuario diferente del original. Cuanto mayor es la especificidad de los activos involucrados en una transacción, mayor es el perjuicio potencial derivado de una conducta oportunista de la contraparte.

6 El propio Williamson, en un trabajo posterior sobre la TCT (Williamson, 1991), reconoció que entre el "continuo" formado por las dos formas puras de gobierno (mercado-jerarquía) existe una gran variedad de estructuras híbridas que emergen en la economía.

7 Para una revisión detallada de este tipo de estudios ver Cohen (1995).

\section{BIBLIOGRAFÍA}

Arrow, K. (1962): "The economic implications of learning by doing", Review of Economic Studies, vol. 29, pp. 155173.

Arora, A. y Gambardella, A. (1994): "Evaluating technological information and utilizing it: Scientific knowledge, technological capability and external linkages in biotechnology", Journal of Economic Behavior and Organization, vol. 24, pp. 91-114.

Arora, A. y Gambardella, A. (1990): "Complementarity and external linkages: The strategies of the large firms in biotechnology", Industrial Economics, vol. 38, pp. 361-379.

Athey, S. y Stern, S. (1998): "An empirical framework for testing theories about complementarity in organizational design", NBER working paper, 6600.

Benavides, C. (1998): Tecnología, innovación y empresa, Madrid, Pirámide.

Belderbos, R.; Carree, M.; Diederen, B.; Lokshin, B. y Veugelers, R. (2004): "Heterogeneity in R\&D cooperation strategies", International Journal of Industrial Organization, vol. 22, pp. 1237-1263.

Beneito, P. (2003): "Choosing among alternative technological strategies: An empirical analysis of formal sources of innovation", Research Policy, vol. 32, pp. 693-713.

Bönte, W. (2003): "R\&D and productivity: Internal vs external R\&D - evidence from West German manufacturing industries", Economics of Innovation and New Technology, vol. 12, pp. 343360.

Cassiman, B. y Veugelers, R. (2006): "In search of complementarity in innovation strategy: internal $R \& D$ and external knowledge acquisition", $M a-$ nagement Science, vol. 52, pp. 68-82.

Chesnais, F. (1996): "Technological agreements, networks and selected issues in economic theory", en R. Coombs, A. Richards, P. Saviotti y V. Walsh (eds.), Technological Collaboration. The Dy- namics of Cooperation in Industrial Innovation, Cheltenham, Edward Elgar.

Cohen, W. M. y Levinthal, D. A. (1990): "Absorptive Capacity: A new perspective on learning and innovation", $A d$ ministrative Science Quarterly, vol. 35, pp. 128-152.

Coombs, R. (1996): "Core competences and the strategic management of $R \& D "$, $R \& D$ Management, vol. 26, pp. 345355.

Croisier, B. (1998): "The governance of external research: Empirical testing of some transaction-cost related factors", $R \& D$ management, vol. 28, pp. 289-298.

Freel, M. (2003): "Sectoral patterns of small firm innovation, networking and proximity", Research Policy, vol. 32, pp. 751-770.

Hagedoorn, J. (2002): "Inter-firm R\&D partnerships: An overview of major trends and patterns since1960", Research Policy, vol. 31, pp. 477-492.

Howells, J. (1999): "Regional systems of innovation?", en D. Archibugi, J. Howells, J. Michie (eds.), Innovation Policy in a Global Economy, Cambridge, Cambridge University Press.

Laursen, K. y Salter, A. (2006): "Open for Innovation: The role of openness in explaining innovative performance among U.K. manufacturing firms", Strategic Management Journal, vol. 27, pp. 131-150.

Lowe, J. y Taylor, P. (1998): "R\&D and technology purchase through licence agreements: Complementarity strategies and complementarity assets", $R \& D$ Management, vol. 28, pp. 263278.

Maidique, M. A. y Zirguer, B. J. (1985): "The new product learning cycle", Research Policy, vol. 14, pp. 299-313.

Mariti, P. y Smiley, R. H. (1983): "Co-operative agreements and the organization 
of industry", Journal of Industrial Economics, vol. 31, pp. 437-451.

Martínez Sánchez, A. (1989): "La gestión estratégica de la tecnologia", Alta Dirección, vol. 146 (julio-agosto), pp. 87-98.

Milgrom, P. y Roberts, J. (1990): "The economics of modern manufacturing: Technology, strategy, and organization", American Economic Review, vol. 80, pp. 511-528.

Miotti, L. y Sachwald, F. (2003): "Co-operative R\&D: why and with whom? An integral framework of analysis", Research Policy, vol. 32, pp. 1481-1499.

Mowery, D. C. (1983): "The relationship between intrafirm and contractual forms of industrial research in American manufacturing, 1900-1940", Exploration in Economics History, vol. 20, pp. 351-374.

Mowery, D. C. y Rosenberg, N. (1989): Technology and the Pursuit of Economic Growth, Cambridge, Cambridge University Press.

Nelson, R. (2000): "National innovation systems", en Z. Acs (ed.), Regional Innovation, Knowledge and Global Change, London, Pinter.
Oerlemans, L.; Meeus, M. y Boekema, F. (1998): "Do networks matter for innovation? The usefulness of the economic network approach in analysing innovation", Tijdschrift voor Economische en Sociale Geografie, vol. 89, pp. 298-309.

Oerlemans, L. y Meeus, M. (2001): "R\&D cooperation in a transaction cost perspective", Review of Industrial Organization, vol. 18, pp. 77-90.

Pisano, G. (1989): "Using equity participation to support exchange: Evidence from the biotechnology industry", Journal of Law, Economics and Organization, vol. 5, p. 109.

Pisano, G. (1990): "The R\&D boundaries of the firm: An empirical analysis", $A d$ ministrative Science Quarterly, vol. 35, p. 153.

Rosenberg, N. (1982): Inside the black box. Technology and economics, Cambridge, Cambridge University Press.

Sakakibara, M. (1997): "Evaluating government-sponsored R\&D consortia in Japan: who benefits and how?", Research Policy, vol. 26, pp. 447-473.

Teece, D. J.; Pisano, G. P. y Shuen, A. (1997): "Dynamic capabilities and strategic management," Strategic Management Journal, vol. 18, pp. 509-533.

Tyler, B. B. y Steensma, H. K. (1995): "Evaluating Technological Collaborative Opportunities: A Cognitive Modelling Perspective", Strategic Management Journal, vol. 16, pp. 43-70.

Veugelers, R. (1997): "Internal R\&D expenditures and external technology sourcing", Research Policy, vol. 26, pp. 303-315.

Veugelers, R. (1998): "Collaboration in R\&D: An Assessment of Theoretical and Empirical Findings", De Economist, vol. 146, pp. 419-443.

Wernerfelt, B. (1984): "A resource-based view of the firm", Strategic Management Journal, vol. 5, pp. 171-180.

Williamson, Oliver E. (1981): "The Economics of Organization: The Transaction Cost Approach", American Journal of Sociology, vol. 87, pp. 548-577.

Williamson, Oliver E. (1985): The Economic Institutions of Capitalism. Firms, Markets, Relational Contracting, New York, The Free Press.

Williamson, Oliver E. (1996): The mechanisms of Governance, 0xford, Oxford University Press. 